\title{
HIGHLIGHTS
}

THYROID GLAND

\section{Hypothyroidism found to be a frequent occurrence after partial thyroidectomy}

The incidence of hypothyroidism after partial thyroidectomy could be at least $10.9 \%$, according to the findings of a retrospective analysis performed by a group from Melbourne, Australia.

Partial thyroidectomy involves the surgical removal of one lobe of the thyroid gland. Although patients who undergo this procedure retain some thyroid function, the incidence and potential risk factors for postoperative hypothyroidism remain unclear. Shirley Su and colleagues have attempted to address this issue by reviewing the medical notes of 294 patients who underwent partial thyroidectomy at the Monash University Endocrine Surgery

Unit between August 1992 and

June 2006.

Indications for partial thyroidectomy included the presence of thyroid nodules with symptoms of hyperthyroidism and suspicion of thyroid carcinoma after fine-needle aspiration biopsy; all of the operations were performed by the same surgeon. Patients with preoperative hypothyroidism were excluded from the analysis.

\section{4 ...these three factors had an $80 \%$ positive predictive value for hypothyroidism 77}

Postoperative hypothyroidism was detected in 32 (10.9\%) of the patients; the mean time to diagnosis was 8.2 months and the mean TSH level was $9.3 \mathrm{mIU} / \mathrm{l}$. Although $84.4 \%$ of patients who developed hypothyroidism had preoperative TSH levels within the normal range (0.5-4.0 $\mathrm{mIU} / \mathrm{l})$, the incidence of postoperative hypothyroidism was greater among patients with preoperative TSH levels of 2.5-4.0 mIU/l than among those with preoperative TSH levels of 0.5-2.4 mIU/1. For the most part, postoperative hypothyroidism was subclinical (78.1\% of all cases), and only patients with overt hypothyroidism received levothyroxine (mean dose $69.4 \mu \mathrm{g}$ daily).

In addition to preoperative TSH levels at the high end of the normal range, patients who developed postoperative hypothyroidism were more likely to have histologically confirmed thyroiditis and detectable antithyroid antibodies than were patients who remained euthyroid. The combination of these three factors had an $80 \%$ positive predictive value for hypothyroidism.

Su et al. conclude that patients undergoing partial thyroidectomy should be closely monitored for the development of postoperative hypothyroidism.

Vicky Heath

Original article Su, S. Y. et al. Hypothyroidism following hemithyroidectomy: a retrospective review. Ann. Surg. 250 991-994 